CASE REPORT

\title{
Chronic compartment syndrome of the forearm in competitive motor cyclists: a report of two cases
}

\author{
J N Goubier, G Saillant
}

Br J Sports Med 2003;37:452-454

Exertional compartment syndrome of the forearm is rare. However, it should be considered in cases of a painful forearm during motorcycle racing. Pressure measurements of all compartments during exercises that simulate the actions of racing confirm the diagnosis. An exertional electromyography may be useful to reveal a nerve compression associated with the compartment syndrome. Fasciotomy of the affected compartments allows relief of symptoms and return to previous activities in all cases.

C hronic exertional compartment syndrome of the lower limbs is well known. Chronic compartment syndrome of the forearm is rare, however, with fewer than 15 cases published to our knowledge..$^{1-7}$ We present a case history of two patients with chronic compartment syndrome of the forearms.

\section{CASE REPORT 1}

A 28 year old professional motorcycle racer complained of pain in the right forearm lasting for several months. This pain only occurred during racing, and was progressively making this activity more difficult. Physical examination showed symmetrical functioning of the superficial and deep flexor compartments of the forearm. There was no sign of nerve compression. Forearm muscles were weak after 15 minutes of handgrip exercises that simulated the movements of racing. A diagnosis of chronic compartment syndrome was evoked.

Nerve conduction velocity was normal. Forearm compartmental pressures were measured with and without muscular activity, by a slit catheter (Howmedica, Mahway, NJ, USA ). Given the results (table 1), compartment syndrome of the palmar compartment was diagnosed.

A fasciotomy of the superficial and deep compartments was performed. The patient returned to his normal activities seven weeks after the operation without any discomfort. Twenty two months later, he presented with the same symptoms in the left forearm. The compartment pressures of the affected forearm were measured before and after exercise (table 1). The deep

Table 1 Compartment pressure measured before and after 15 minutes of handgrip exercises $(\mathrm{mm} \mathrm{Hg})$

\begin{tabular}{lllllll}
\hline & \multicolumn{2}{l}{ Patient 1} & & \multicolumn{2}{l}{ Patient 2 } \\
\cline { 2 - 3 } \cline { 6 - 7 } \cline { 5 - 6 } & Right & Left & & Right & Left \\
\hline Before exercises & 15 & 23 & & 17 & 15 \\
After 15 min of exercises & 34 & 36 & & 33 & 19 \\
15 min after exercises & 31 & 32 & & 31 & 15 \\
30 min after exercises & 14.5 & 22 & & 17.5 & 15 \\
\hline
\end{tabular}

and superficial compartments were surgically decompressed. As with the right forearm, he returned to competition without any pain or weakness of his forearm.

\section{CASE REPORT 2}

A 25 year old motorcycle racer presented with circumferential pain in the right forearm and weakness of grip during racing. Physical examination failed to reveal any loss of motor or sensibility, and there was no sign of vascular problems. No symptoms of forearm muscle tendinitis were present. Exertional nerve conduction velocities were normal. Forearm compartment pressures were measured before and after grip exercises (table 1). The diagnosis of chronic exertional compartment syndrome of the right forearm was confirmed, and the anterior compartments were surgically decompressed. Three months after fasciotomy, the patient returned to his previous level of activity symptom free.

\section{DISCUSSION}

The anatomical conditions of chronic compartment syndrome in the lower limb have been well described in the literature. ${ }^{3}$ In the forearm, the volar and dorsal compartments are interconnected, unlike the leg compartments. Several anatomical structures, however, limit the dorsal and volar compartments. ${ }^{3}$ The interosseous membrane, the ulna, and the radius separate the volar and dorsal compartments. The anti-brachial fascia limits anteriorly the volar compartment and posteriorly the dorsal compartment.

The thick aponeurosis of the flexor digitorum profundus separates one superficial and one deep volar compartment. The median and ulnar nerves are placed respectively laterally and medially on the flexor digitorum profundus aponeurosis. However, interconnections between the superficial and deep volar compartments are not sufficient to prevent a large increase in pressure during continuous contraction of the flexor muscles. Therefore chronic compartment syndrome may occur in motorcycle racing activities in which flexor muscle contraction is continuous to control the brake lever and keep the motor bike on the road.'

The diagnosis of compartment syndrome of the forearm muscles is confirmed by the measurement of forearm compartmental pressure. All compartments (superficial and deep volar compartments and dorsal compartments) must be measured. ${ }^{6}$ For the volar compartments, the catheter is introduced into the proximal third of the forearm on the middle line. ${ }^{8}$ The catheter is inserted at an angle of $30^{\circ}$ to the medial side and superficially to measure the superficial compartment. Then, it is gently introduced in the same direction to measure the deep compartment. These measurements must be performed before and during exercises that simulate the actions used in racing and then after 15 and 30 minutes of rest. ${ }^{1}$ However, it is difficult to decide on a normal value of intracompartmental pressure during exercise. This value 


\section{Take home message}

Chronic exertional compartment syndrome of the forearm is rare. Clinical examination, pressure measurement, and electromyography confirm the diagnosis. Treatment consists of fasciotomy of the affected compartments which allows return to previous activities.

depends on the timing of the measurement and varies from 40 $\mathrm{mm} \mathrm{Hg}$ after 30 minutes of exercise in a badly affected patient reported by Kutz et $\mathrm{al}^{4}$ to $90 \mathrm{~mm} \mathrm{Hg}$ under maximal contraction in a symptom-free volunteer reported by Berlemann et al. ${ }^{2}$ Therefore the diagnosis of compartment syndrome may be evoked if the pressure under maximal contraction is high and principally if there is a slow return to the baseline level.

Other examinations have been described to obtain the diagnosis of compartment syndrome. Amendola et a $l^{9}$ demonstrated the potential value of magnetic resonance imaging in the diagnosis of compartment syndrome with changes in compartments after exercise, in $\mathrm{Tl}$ weighted images. However, the importance of pressure level and the return to baseline pressure cannot be evaluated with magnetic resonance imaging. Therefore, it is not currently used for routine analysis of exertional compartment syndrome. Thallium-201 singlephoton emission tomography imaging has been performed to confirm the diagnosis of chronic compartment syndrome, suggesting the role of a perfusion deficit in the affected compartment. ${ }^{10}$ However, for Trease et $a l^{11}$, this method does not contribute to the diagnosis of exertional compartment syndrome.

Several diagnoses have to be considered in the case of pain of the forearm during exercise. Clinical examination may eliminate common tendinitis of the forearm muscles and brachial artery compression. ${ }^{12}$ Nerve compression (median nerve, anterior interosseous nerve, or ulnar nerve) may simulate and be associated with compartment syndrome. ${ }^{2}$ Therefore exertional electromyography must be performed to eliminate nerve compression. As for pressure measurement, nerve conduction must be evaluated before, during, and after the period of stress. Nerve conduction velocity less than $50 \mathrm{~m} / \mathrm{s}$ is considered slow and may indicate a surgical release of the affected nerve. ${ }^{4}$

The treatment for exertional compartment syndrome in the forearm is fasciotomy of all affected compartments. This treatment has been successful in all publications, ${ }^{1-7}$ and no recurrences have been described. Therefore fasciotomy is sufficient in forearm compartments, in contrast with leg compartments, in which fasciectomy may be necessary. ${ }^{13}$ The technique of volar fasciotomy has been described by Henry cited by Allen and Barnes': with an anterior approach, an incision of anti-brachial fascia and flexor digitorum superficialis aponeurosis is performed. Then, flexor digitorum superficialis is retracted laterally, the ulnar artery and nerve are gently placed on the medial side, and the median nerve on the lateral side, and the flexor digitorum profundus aponeurosis is incised. Finally, nerve decompression may be useful if the exertional electromyography shows a slow nerve conduction velocity.

\section{Authors' affiliations}

J N Goubier, Service de Chirurgie Orthopédique et Traumatologique, Chirurgie Nerveuse Périphérique, Chirurgie de la Main, Groupe Hospitalier Bichat-Claude Bernard, 46 rue Henri Huchard, 75877 Paris Cédex 18, France
G Saillant, Service de Chirurgie Orthopédique, Traumatologique et Réparatrice de l'Appareil Locomoteur, Groupe Hospitalier Pitié-Salpétrière, 83 boulevard de I'hôpital 75651 Paris Cédex 13, France

Correspondence to: Mr Goubier, Service de Chirurgie Orthopédique et Traumatologique, Chirurgie Nerveuse Périphérique, Chirurgie de la Main, Groupe Hospitalier Bichat-Claude Bernard, 46 rue Henri Huchard, 75877 Paris Cédex 18, France; goubjnal@club-internet.fr

Accepted 25 September 2002

\section{REFERENCES}

1 Allen MJ, Barnes MR. Chronic compartment syndrome of the flexor muscles in the forearm: a case report. J Hand Surg [Br] 1989;14:47-8.

2 Berlemann U, al-Momani Z, Hertel R. Exercise-induced compartment syndrome in the flexor-pronator muscle group. A case report and pressure measurements in volunteers. Am J Sports Med 1998;26:439-41.

3 Kouvalchouk JF, Watin Augouard L, Dufour O, et al. Chronic stress-related compartment syndrome of the forearm. Rev Chir Orthop Reparatrice Appar Mot 1993;79:351-6.

4 Kutz JE, Singer R, Lindsay M. Chronic exertional compartment syndrome of the forearm: a case report. J Hand Surg [Am] $1985 ; 10: 302-4$

5 Soderberg TA. Bilateral chronic compartment syndrome in the forearm and the hand. J Bone Joint Surg [Br] 1996;78:780-2.

6 Rydholm U, Werner C, Ohlin P. Intracompartimental forearm pressure during rest and exercise. Clin Orthop 1983;175:213-15.

7 Pedowitz RA, Toutounghi FM. Chronic exertional compartment syndrome of the forearm flexor muscles. J Hand Surg [Am] 1988;13:694-6.

8 Gelberman R, Garfin S, Hergenroeder P, et al. Compartment syndromes of the forearm: diagnosis and treatment. Clin Orthop $1981 ; 161: 252-61$

9 Amendola A, Rorabeck CH, Vellett D, et al. The use of magnetic resonance imaging in exertional compartment syndromes. Am J Sports Med 1990;18:29-34

10 Takebayashi S, Takazawa H, Sasaki R, et al. Chronic exertional compartment syndrome in lower legs: localization and follow-up with thallium-201 SPECT imaging. J Nucl Med 1997;38:972-6.

11 Trease L, van Every B, Bennell K, et al. A prospective blinded evaluation of exercise thallium-201 SPET in patients with suspected chronic exertional compartment syndrome of the leg. Eur J Nucl Med 2001;28:688-95

12 Bassett F, Spinner R, Schroeter T. Brachial artery compression by the lacertus fibrosus. Clin Orthop 1994;307:110-16.

13 Slimmon D, Bennell K, Brukner P, et al. Long-term outcome of fasciotomy with partial fasciectomy for chronic exertional compartment syndrome of the lower leg. Am J Sports Med 2002;30:581-8.

\section{COMMENTARIES}

The diagnosis of chronic exertional compartment syndrome of the forearm flexor muscles is difficult indeed. Although the diagnosis can be suspected clinically, unfortunately, at the current time it cannot be established without relying on results from intracompartmental pressures. Therein remain further difficulties. The diagnosis must be either inferred from limited data in isolated case reports dealing with compartment syndromes of the upper limb or extrapolated from criteria proposed for those of the leg. ${ }^{1}$

In this regard, values for normals need to be established, ${ }^{23}$ and abnormal values need to be defined, including pressures before exercise (resting baseline), during exercise (for example, mean muscle, maximal muscle contraction, and muscle relaxation pressures), and after exercise (such as one minute and five minute pressures, and time to normalisation). Postoperative values can also be compared with preoperative ones and contrasted with contralateral values (although both bilateral symptomatic compartment syndromes and subclinical elevated pressures may occur).

This report of two patients with chronic exertional compartment syndrome in the forearm is important for several reasons. It provides further information on the causes-that is, repetitive, often continuous, resisted activities-and potentially at risk avocations-for example, motor cycle racers. ${ }^{4}$ In addition, it serves as a gentle reminder to clinicians to suspect this diagnosis in patients with episodic, 
or exercise related symptoms. Finally, it highlights our limitations in diagnosing this condition and the importance in improving this.

R J Spinner

Mayo Clinic, Department of Neurologic Surgery and Ortho, 200 First Street, SW Rochester, MN 55905, USA; Spinner.Robert@mayo.edu

\section{REFERENCES}

1 Pedowitz RA, Hargens AR, Mubarak SJ, et al. Modified criteria for the objective diagnosis of chronic compartment syndrome of the leg. Am J Sports Med 1990:18:35-40.

2 Berlemann U, Al-Momani Z, Hertel R. Exercise-induced compartment syndrome in the flexor-pronator group. A case report and pressure measurements in volunteers. Am J Sports Med 1998;26:439-41.

3 Rydholm U, Werner C-O, Ohlin P. Intracompartmental forearm pressure during rest and exercise. Clin Orthop 1983;175:213-15.

4 Allen MJ, Barnes MR. Chronic compartment syndrome of the flexor muscles in the forearm: a case report. J Hand Surg [Br] 1989;14:47-8.

The authors should be thanked for presenting this work because, if chronic anterior compartment syndrome of the forearm has the same pathophysiology as the leg, this knowledge is more recent and its incidence is underestimated. It is seen not only in sports that require strong and prolonged contraction of the flexor muscles of the fingers (motorcycling and windsurfing) but also in manual workers (one case out of six in our series). The authors have reason to insist on the measurement of pressure for diagnosis, but isotope scintigraphy should be added. It shows a delay in the arrival of tracer on the painful side and, a few minutes later, a reversal of the scan, which increases on the abnormal side, confirming the presence of stasis; the crossing of the two lines is significant. The diagnosis has to be certain, because there are other causes of forearm pain in sport that should be considered.

Like the authors, we think that the treatment must be surgical. However, because of neurological risks and the anatomy of the forearm, the subcutaneous approach is not indicated here. In addition, we think that a partial fasciectomy should be used rather than a fasciotomy. The following follow up procedures should be carried out: drainage of the compartments, ice, and early muscle work to prevent amyotrophy with the risk of aponeurotic, potentially stenotic, scars.

Correct diagnosis, a complete technical procedure, and careful follow up should give excellent results.

J-F Kouvalchouk Hôpital Foch, Service de Chirurgie Orthopédique et de Traumatologie du Sport, 40, rue Worth, 92150 Suresnes, France; jf.kouvalchouk@hopital-foch.org 